\title{
Lubricated Friction and the Hersey Number
}

\author{
B. Veltkamp, ${ }^{1}$ K. P. Velikov, ${ }^{1,2}$ C. H. Venner, ${ }^{3}$ and Daniel Bonn ${ }^{1}$ \\ ${ }^{1}$ Van der Waals-Zeeman Institute, Institute of Physics, University of Amsterdam, 1098XH Amsterdam, The Netherlands \\ ${ }^{2}$ Unilever Innovation Centre Wageningen, Bronland 14, $6708 \mathrm{WH}$ Wageningen, The Netherlands \\ ${ }^{3}$ University of Twente, De Horst 2, 7522LW Enschede, The Netherlands
}

(Received 14 September 2020; revised 15 December 2020; accepted 22 December 2020; published 26 January 2021)

\begin{abstract}
Most frictional contacts are lubricated in some way, but is has proven difficult to measure and predict lubrication layer thicknesses and assess how they influence friction at the same time. Here we study the problem of rigid-isoviscous lubrication between a plate and a sphere, both experimentally and theoretically. The liquid layer thickness is measured by a novel method using inductive sensing, while the friction is measured simultaneously. The measured values of the layer thickness and friction on the disk are well described by the hydrodynamic description of liquid flowing through a contact area. This allows us to propose a modified version of the Hersey number that compares viscous to normal forces and allows us to rescale data for different geometries and systems. The modification overcomes the shortcomings of the commonly used Hersey number, adds the effects of the geometry of the configuration on the friction, and successfully predicts the lubrication layer thickness.
\end{abstract}

DOI: 10.1103/PhysRevLett.126.044301

Lubricated friction is a phenomenon that occurs in a large variety of different fields. Engineers often opt to use oils that smoothen the movement of the rigid internal parts like gears, bearings, or chains to reduce energy consumption and lengthen the lifetime of the machinery. Lubrication can cause the soft rubber wheels of cars to slip in snowy or rainy circumstances. Similarly, the steel wheels of trains losing grip due to leaves on the rails is a costly problem [1]. In the food and cosmetics industry, lubricated friction plays a large role in the sensory perception in the human mouth or on human skin [2]. Even in ancient Egypt people knew how to reduce friction, which can be concluded from them spreading water in front of heavy sculptures, making it easier to pull them through the desert sand [3].

It is estimated that $20 \%-30 \%$ of the world's energy consumption is related to friction and wear [4]. Therefore, minimizing friction is an important goal in many engineering problems that involve sliding or rotating parts. In the vast majority of cases, this is achieved by lubricating the contacts. A full physical understanding of a lubricated contact requires an experimentally verified theory describing the liquid layer thickness as well as the frictional force. Much work has been performed on both aspects, yet unification of the two theories into one single experiment has proven to be difficult.

On the one hand, theoretical hydrodynamic investigations for the layer thickness are plentiful, with descriptions that take into account other effects such as elastically deformable surfaces $[5,6]$ and pressure-viscosity relations [6-8]. However, even for the simplest case of hard surfaces and isoviscous liquids, the unification of layer thickness and friction in a single theory has not been experimentally confirmed.

On the other hand, it is found that friction for lubricated contacts is qualitatively described by a Stribeck curve, which explains how friction scales with the relevant physical parameters in the boundary, mixed, and hydrodynamic lubrication regimes $[5,9,10]$. The curve relates the friction coefficient to a dimensionless parameter, called the Hersey number, which should consist of a combination of all relevant physical parameters in the configuration. Empirically, it has been found that, in the hydrodynamic regime, the friction coefficient $\mu$ (the ratio of friction force and normal force) scales with the "conventional" Hersey number, defined as $H r \equiv\left(\eta U_{0} / F_{N}\right)$, where $\eta, U_{0}$, and $F_{N}$ are the viscosity, sliding velocity, and normal force, respectively. The scaling has been suggested to be linear [5,11], although more recent work uses a power-law relation [12-15].

However, this Hersey number is not sufficient for a full physical understanding for several reasons. First, this combination of parameters has dimension $\mathrm{m}^{-1}$, so it is not dimensionless. Others have worked around this problem by introducing a constant length scale into the Hersey number, for example, the surface roughness [14] or radius of curvature [16]. Obviously, adding an extra constant factor will keep the proportionality properties, but does not explain how friction depends on the geometry of a sliding experiment. Second, this Hersey number does not contain the liquid layer thickness between the sliding contacts, so it is impossible to determine when this thickness becomes so small that the mixed lubrication regime is reached. Finally, relating to our first point, there is no direct relation between 
Hersey number and friction, as previous research has only investigated the proportionality, making an accurate prediction prior to an experiment impossible. This has forced researchers to perform cumbersome series of sliding experiments to obtain a separate Stribeck curve for each slightly different configuration $[11,15]$. On small scales, techniques such as the surface force apparatus [17] or atomic force microscopy [18] allow one to perform very precise friction and lubrication experiments. These techniques investigate the microscopic origins of friction, rather than providing a practical description of friction that typically occurs on larger length scales and can be used for engineering purposes.

Here, we use a novel method of inductive sensing to measure the layer thickness between two hard surfaces, which, combined with simultaneous friction measurement, allows us to test conventional lubrication theory for both layer thickness and friction. The results naturally lead to the proposal of a modified Hersey number, solving the aforementioned problems with the conventional Hersey number.

The experimental setup consists of a glass plate on which aluminum disks (radius $R_{s}=1.95 \mathrm{~cm}$ ) are pulled along a circular track (radius $10 \mathrm{~cm}$ ) in between two Plexiglas walls that confine the lubricant to the track [see Fig. 2 in the supplementary materials for details]. The disks have a spherical bottom with radius of curvature $R=100 \mathrm{~cm}$ (the flattest disk) and $R=25 \mathrm{~cm}$ (the most curved disk). The rotation is performed by an arm connected to a rheometer head (Anton Paar rheometer: MCR 702 multidrive), allowing for control of the pulling speed, while measuring the torque and hence the friction. Error in the friction measurements is $5 \mathrm{mN}$, arising from the unconventional usage of the rheometer through the attachment of a long arm. The disk is attached with a flexible thin wire to the far end of the arm, so that the arm itself does not cause any additional normal force being applied onto the disk. Surface roughness of the disk is measured by a profilometer (Keyence Laser Microscope: 3D laser scanning confocal microscope VK-X1000) and found to be $\sim 1 \mu \mathrm{m}$, much smaller than the typical layer thicknesses measured in the experiments and orders of magnitude smaller than the radius of curvature. Adding mass centrally on top of the disk allows for varying normal forces.

The layer thickness is defined as the minimum separation of the two surfaces (Fig. 1). For the flattest disk, this liquid layer thickness is measured by inductive sensing (Texas Instruments, LDC1614EVM evaluation module for inductance to digital convertor). Eight identical sensors are glued underneath the circular $1.1 \mathrm{~mm}$ thick glass track, at regular intervals of $45^{\circ}$. Calibration consists of two steps. First, before positioning of the sensors, a gauge is performed by fixing a sensor on a flat plate and moving the disk toward it to relate the change of height to the change of inductance. Second, pulling the disk along the track without a liquid yields the inductance value for a layer thickness $h_{0}=0$.

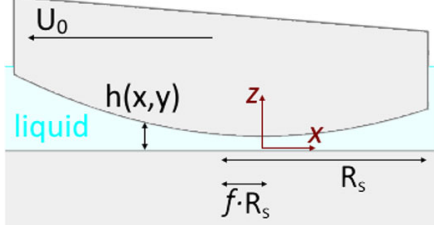

FIG. 1. Schematic of the configuration of the fully lubricated contact between a flat surface and a slightly curved, tilted disk. The disk moves with velocity $U_{0}$ in the negative $x$ direction, indicated by the arrow, and is tilted such that the narrowest part of the gap $h(x, y)=h_{0}$ is shifted in positive $x$ direction by an amount $f R_{s}$.

This allows one to measure the layer thickness $h_{0}$ when a lubricated disk moves over the sensor. Repeating this procedure with various sensors yields identical gauges within $5 \mu \mathrm{m}$, which is sufficiently accurate for testing our hydrodynamic lubrication theory.

The disk is lubricated by pouring one of two types of polydimethylsiloxane oil onto the track at room temperature. The viscosity of the tested oils is obtained by rheology [19], yielding $\eta=53.5$ and $104 \mathrm{mPas}$. The experimental protocol consists of pouring the liquid, then letting the disk rotate with $4 \mathrm{~cm} / \mathrm{s}$ to evenly distribute the liquid, and finally increasing the velocity ramping from 2 to $10 \mathrm{~cm} / \mathrm{s}$ in $240 \mathrm{~s}$. The amount of liquid is chosen such that the front of the disk is fully lubricated during the entirety of the measurement. For some measurements, a control experiment is performed where the velocity ramp is inverted. This yields the same result for the layer thickness measurement within the $5 \mu \mathrm{m}$ error, so that it can be concluded that a steady state is reached.

Fig. 2 shows the liquid layer thickness and friction as a function of velocity and normal force. The lines represent theoretical predictions, of which the derivation follows below. There is quantitative agreement between theory and experiment for the layer thickness, without any adjustable parameter. For the friction, our model only takes into account the friction arising from underneath the disk, yet in our experiments additional friction sources were present: mainly the ploughing of the disk through the liquid and friction with the side walls of the channel. To account for this, we add a friction component to all of the calculated friction forces (black dashed lines in Fig. 2). We find that a very satisfactory description of our data is obtained when we use a constant background noise term plus a term that scales linearly with viscosity, velocity, and the frontal area of the disk to compensate the ploughing, thus giving us two tunable parameters to fit the entirety of our data. With this relatively small correction, the normal force and velocity dependence of the friction is also quantitatively accounted for, giving us some confidence in the hydrodynamic description detailed below.

Our model tends to underestimate the measured friction for relatively high normal force and low velocity. This can 

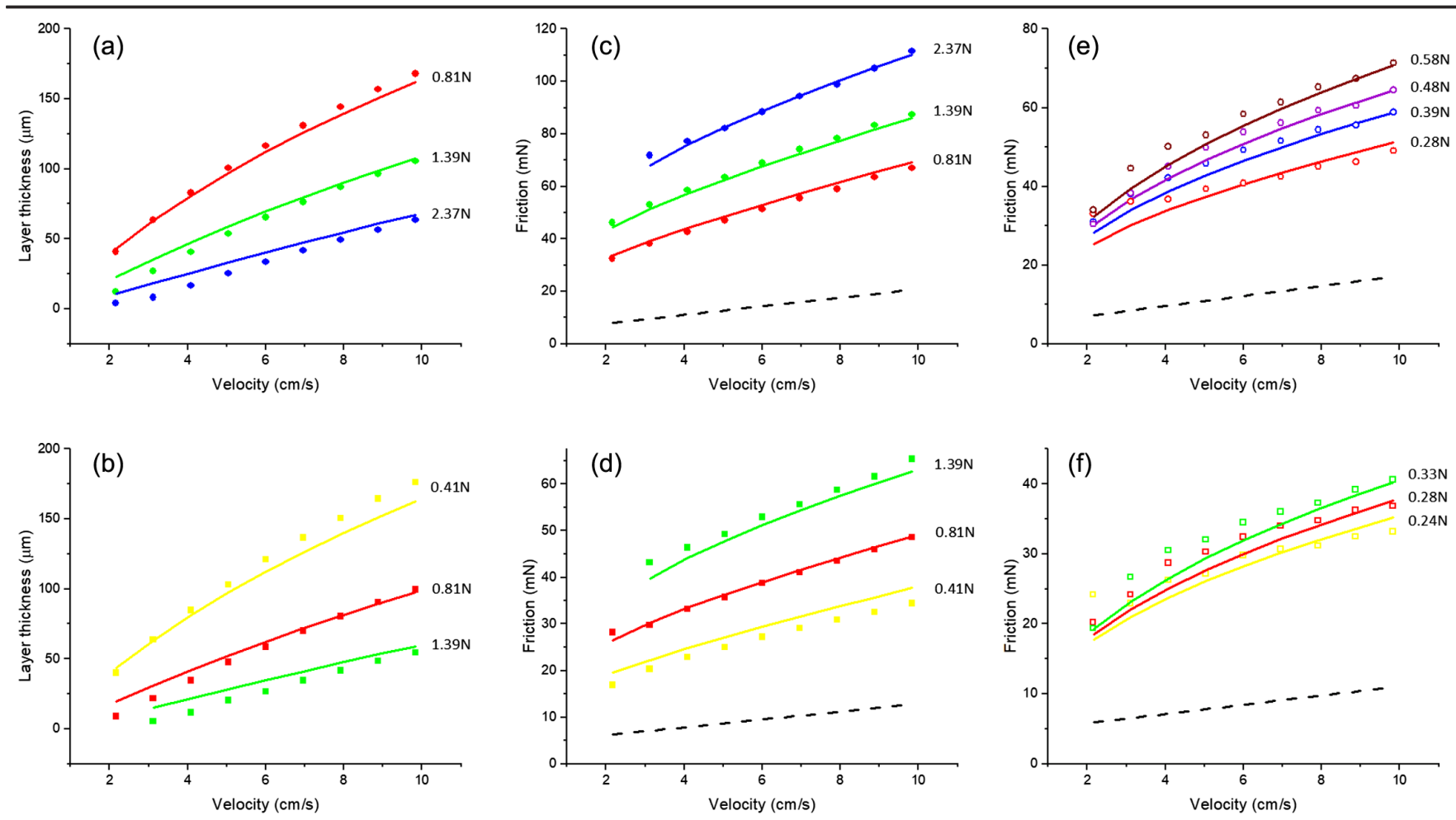

FIG. 2. Measurements of layer thickness (a),(b) and friction force (c)-(f) as a function of velocity and normal force, with (a),(c), (e) $104 \mathrm{mPa} s$ and (b),(d),(f) $53.5 \mathrm{mPa} s$. (a)-(d) Filled symbols denote a disk with radius of curvature $R=100 \mathrm{~cm}$; (e),(f) open symbols denote a disk with $R=25 \mathrm{~cm}$. All measurements use a disk with radius $R_{s}=2.0 \mathrm{~cm}$. Values on the right-hand side indicate total normal force on the disk. Solid lines correspond to theoretical predictions, Eqs. (3) and (5). For the friction measurements, an additional noise and ploughing friction (black dashed lines) from sources other than the lubricated contact is postulated to match theory and experiments. Some measurements do not reach the lowest velocities (as can be seen by the abrupt stoppage of the data), because the low layer thickness results in stick-slip behavior. Error on the layer thickness measurements is $5 \mu$ m. (The lowest data points are therefore indistinguishable from a layer thickness of zero and the transition to the mixed regime is likely close).

be ascribed to the layer thickness being so small that surface effects start to play a role. We are uncertain about the precise physical origin of the excess force measured. It could be that the intimate contact yields an adhesive van der Waals force. However, we cannot exclude the possibility that tiny dust or dirt particles, with size equal to the layer thickness, enter the contact and disturb the measurement, which would explain the inconsistent nature of stick-slip events. In any case, it is worth noting that the presence of excess force at low layer thickness could be an indication that the mixed regime is being entered.

To model the friction, we consider a disk with radius $R_{s}$ and a spherical bottom with radius $R$, sliding with velocity $U_{0}$ in the negative $x$ direction on a flat surface (see Fig. 1), with its inlet fully emerged in liquid. Having only this segment of a sphere instead of a full sphere, is mathematically identical to what is known in literature as starved lubrication [5]. The gap between both surfaces is given by a parabolic approximation $h=h_{0}+\left[\left(x^{2}+y^{2}\right) / 2 R\right]$, with $h_{0}$ the lowest point and $R$ the radius of curvature.

For $h_{0} \ll R_{S}$ the lubrication approximation yields a solution of the Stokes equations in the form of the Reynolds equation $[6,20]$, which is a differential equation for the pressure distribution $p=p(x, y)$ in the region of contact,

$$
\frac{\partial}{\partial x}\left(\frac{h^{3}}{12 \eta} \frac{\partial p}{\partial x}\right)+\frac{\partial}{\partial y}\left(\frac{h^{3}}{12 \eta} \frac{\partial p}{\partial y}\right)=\frac{\partial}{\partial x}\left(\frac{1}{2} h U_{0}\right)
$$

where $\eta$ is the dynamic viscosity of a Newtonian liquid.

In the case of a line contact, when all $y$ dependence drops out, an exact solution for $p$ can be found [7]. However, with the $y$ terms included and a noncentrally positioned origin, this is no longer the case, and a numerical approach is required. A similar problem with a sliding ball instead of a disk, effectively taking the limit $R_{s} \rightarrow R$, has been studied extensively by Kapitza [21], and later Brewe et al. [22]. (In this limit, the parabolic approximation is no longer valid near the edge of the disk, but the edge has no significant contribution to the generated lift, so we expect this limit to predict correct results anyway.) A more general solution with the additional parameter $R_{s}$, to take into account the proximity of the edge of the disk, is derived in the Supplemental Material [23]. This yields the following relations for the normal force $F_{N}$ and the friction force $F_{w}$ on the disk: 

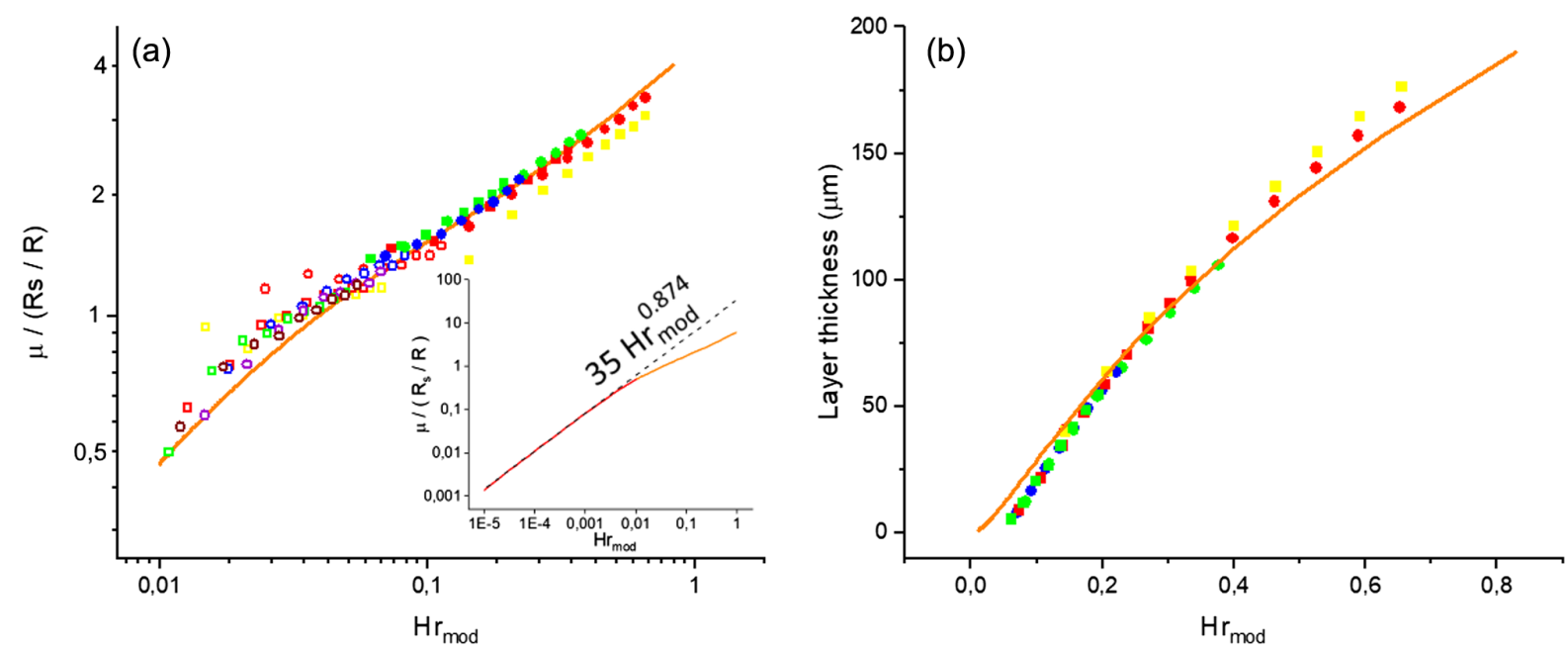

FIG. 3. Measured friction coefficient $\mu$ scaled by (a) $R_{s} / R$ and (b) layer thickness $h_{0}$ as a function of the modified Hersey number calculated by Eq. (4). Symbol shapes and colors correspond to different viscosities, normal forces, and radii of curvature in the same way as in Fig. 2. Theoretical predictions (lines) are calculated by Eqs. (5) and (6). Inset in (a) shows the extension of the theoretical curve of the main figure to low Hersey numbers (red line), which is well approximated by a power law (black dashed line).

$$
\begin{gathered}
F_{N}=\frac{8 \eta U_{0} R^{2}}{R_{s}} \Lambda\left(\tilde{R}_{s}\right), \\
F_{W}=2 \eta U_{0} R K\left(\tilde{R}_{s}\right),
\end{gathered}
$$

where $\Lambda\left(\tilde{R}_{s}\right)$ and $K\left(\tilde{R}_{s}\right)$ are two dimensionless functions that depend on the dimensionless parameter $\tilde{R}_{s}=$ $R_{s} / \sqrt{2 R h_{0}}$. In a steady-state configuration, the load is equal to the normal force, and Eq. (2) can be inverted to find $\tilde{R}_{s}$; hence also the layer thickness. This value is substituted into Eq. (3) to find the friction as a function of normal force. Naturally, a dimensionless number is generated in this process, which we define as the modified Hersey number,

$$
H r_{\text {mod }} \equiv \frac{\eta U_{0} R^{2}}{F_{N} R_{S}}
$$

This results in the formulas for layer thickness and the friction coefficient becoming

$$
\begin{gathered}
h_{0}=\frac{R_{s}^{2}}{2 R}\left(\frac{1}{\Lambda^{-1}\left[1 /\left(8 H r_{\text {mod }}\right)\right]}\right)^{2}, \\
\mu \equiv \frac{F_{w}}{F_{N}}=2 \frac{R_{s}}{R} H r_{\text {mod }} K\left\{\Lambda^{-1}\left[1 /\left(8 H r_{\text {mod }}\right)\right]\right\} .
\end{gathered}
$$

Note that this modified Hersey number differs from the conventional Hersey number, as it depends on the geometry of the configuration (in terms of the curvature and size of the disk), while it maintains the same proportionality with respect to viscosity, velocity, and normal force. The friction coefficient depends in a nonlinear fashion on
Hersey number, due to its dependence on the dimensionless functions $\Lambda^{-1}$ and $K$.

Figure 3 shows Eqs. (5) and (6), along with the same data points as Fig. 2 (with "noise" and "ploughing" friction compensated). In total, four parameters are changed experimentally (viscosity, velocity, normal force, and radius of curvature), leading to a modified Hersey number varying almost 2 orders of magnitude. Our model is not limited to only this range of parameters, but our experimental setup has not allowed us to test beyond this range, mainly due to the proximity of the mixed regime (low Hershey) and the too large forces involved (high Hershey number). We expect the model to work for any combination of parameters that yields a layer thickness large enough that surface effects can be excluded. The geometry of the configuration manifests itself in the modified Hershey number by the scaling $R^{2} / R_{s}$, as a direct consequence of the curved surface being approximated as a parabola. As long as this approximation holds, the scaling holds, and if the contact were to be shaped differently, the scaling would break down as a result of the parameter $R$ being ill defined.

There are two important limits to be considered. First, for many applications, a sliding or rotating sphere is considered, instead of a spherical segment. Here, the limit $R_{s} \rightarrow R$ applies, without any tilt parameter. In this case, the modified Hersey number would take the shape $H r_{\text {mod }}=$ $\left(\eta U_{0} R / F_{N}\right)$, which is a form that has been used by others before [16], as discussed in the Introduction. In practice, the lubrication approximation breaks down near the edge of the disk. However, as the dominant contributions to the lift and friction originate in the region of close contact and not near the edge, we expect that qualitatively our analysis predicts correct trends for this limit. For a microscopic layer thickness and macroscopic radius of curvature, this limit 
corresponds to the configuration where $\tilde{R}_{S} \gg 1$, where $\Lambda\left(\tilde{R}_{s}\right)$ scales linearly with $\tilde{R}_{s}$ [22]. We find that $\Lambda\left(\tilde{R}_{s}\right) \approx$ $0.75 \tilde{R}_{s}$ describes the trend well, although Brewe et al. find $\Lambda\left(\tilde{R}_{s}\right) \approx 1.039 \tilde{R}_{s}$ [24]. We do not know the cause of this discrepancy. Using the former relation to calculate the friction coefficient by Eq. (6) shows that, for $H r_{\text {mod }}<0.01$, the friction coefficient scales with $H r_{\text {mod }}^{0.874}$, which is almost linear [Fig. 3(a)]. This explains why a linear relation in the hydrodynamic regime is often assumed, but also why sometimes a power-law relation is found.

Second, in the limit where the liquid layer thickness goes to zero, a transition between mixed and hydrodynamic lubrication takes place. For a general surface roughness $[5,25]$, the mixed regime is assumed to be when the layer thickness approaches the same value as the root mean square $\sigma$ of the height distribution of surface roughness (under the rigid-isoviscous assumption). Rewriting Eq. (5), putting $h_{0}=\sigma$ and defining $H r_{\bmod }^{M \rightarrow H}$ as the modified Hersey number for when the transition between the mixed and hydrodynamic regimes takes place, the following relation is found:

$$
H r_{\bmod }^{M \rightarrow H}=\frac{1}{8 \Lambda\left(\frac{R_{s}}{\sqrt{2 R \sigma}}\right)} .
$$

There is no universal modified Hersey number that describes the transition, but the transition is geometry dependent through $R_{s}$ and $R$. For curvier surfaces, the mixed regime is entered at lower modified Hersey number than for flatter surfaces. Taking the limit $R_{s} \rightarrow R$ here, and using the approximation for $\Lambda$, yields an approximation for $H r_{\text {mod }}^{M \rightarrow H}$ for a sliding sphere,

$$
H r_{\text {mod }}^{M \rightarrow H}=0.236 \sqrt{\sigma / R} .
$$

This means that, to reduce friction in a sliding contact with known radius of curvature and surface roughness, the contact can be engineered in such a way that $\mathrm{Hr} \gg H r_{\text {mod }}^{M \rightarrow H}$ always holds, so that the contact always remains in the hydrodynamic lubrication regime, for example, by choosing the viscosity of the lubricant accordingly.

Our model only describes the motion of rigid surfaces, which could already be useful in lowly loaded contacts. However, it can also serve as starting point for more advanced calculations, while maintaining the practical form of the friction coefficient being dependent on a simple dimensionless parameter. The model can easily be adjusted for the case of rolling friction by excluding the Couette term in the integral over shear stress. This would change the numerical value of the $K\left(\tilde{R}_{s}\right)$ function, but the shape of Eq. (6) would be conserved. The modified Hersey number can be extended to also include liquids with shear thinning or thickening properties, though the calculation of the dimensionless functions $K\left(\tilde{R}_{S}\right)$ and $\Lambda\left(\tilde{R}_{S}\right)$ will be mathematically more cumbersome. There might even be possibilities of extending the model to deformable surfaces as well by including the Young's modulus of the materials into the modified Hersey number too. The formation of the liquid film in this case has already been theorized and experimentally validated $[5,8]$, yet a similar result for the friction is still missing. Similarly, if the effects occurring at low layer thickness are properly understood, the mixed regime could also be included into the model as well, leading to a more fundamental understanding of the Stribeck curve.

In conclusion, we define a new, modified Hersey number that is dimensionless and allows one to predict friction and lubrication layer thicknesses from the macroscopic quantities in a lubricated sliding experiment. The modified Hersey number and the friction coefficient are directly related to each other, in contrast to the conventional Hersey number that merely describes how friction scales with certain parameters. In addition, the transitions between the different frictional regimes are shown to also be governed by the modified Hersey number. This adds to a more detailed and quantitative understanding of lubricated friction for hard surfaces and the transition to the mixed lubrication regime, a problem of considerable importance in the engineering of friction.

[1] K. Ishizaka, S. R. Lewis, and R. Lewis, The low adhesion problem due to leaf contamination in the wheel/rail contact: Bonding and low adhesion mechanisms, Wear 378-379, 183 (2017).

[2] J. L. Kokini, The physical basis of liquid food texture and texture-taste interactions, J. Food Eng. 6, 51 (1987).

[3] A. Fall, B. Weber, M. Pakpour, N. Lenoir, N. Shahidzadeh, J. Fiscina, C. Wagner, and D. Bonn Sliding Friction on Wet and Dry Sand, Phys. Rev. Lett. 112, 175502 (2014).

[4] K. Holmberg and A. Erdemir, Global impact of friction on energy consumption, economy and environment, FME Trans. 43, 181 (2015).

[5] B. J. Hamrock, S. R. Schmid, and B. O. Jacobson, Fundamentals of Fluid Film Lubrication (Marcel Dekker, New York, 2004).

[6] C.H. Venner and A. Lubrecht, Multilevel Methods in Lubrication (Elsevier, Amsterdam, 2000).

[7] A. E. Cook, Some asymptotic results in hydrodynamic lubrication theory, Appl. Math. Model. 9, 72 (1985).

[8] R. K. Kunz and W. O. Winer, Discussion: Isothermal elastohydrodynamic lubrication of point contacts: Part IIIFully flooded results, J. Lubr. Technol. 99, 275 (1977).

[9] M. D. Hersey, The laws of lubrication of horizontal journal bearings, J. Wash. Acad. Sci. 4, 542 (1914).

[10] Y. Zhang et al., Prediction of the Stribeck curve under full-film elastohydrodynamic lubrication, Tribol. Int. 149, 105569 (2020).

[11] B. Jacobson, The Stribeck memorial lecture, Tribol. Int. 36, 781 (2003). 
[12] E. Hansen, B. Frohnapfel, and A. Codrignani, Sensitivity of the Stribeck curve to the pin geometry of a pin-on-disc tribometer, Tribol. Int. 151, 106488 (2020).

[13] K. Pondicherry, F. Rummel, and J. Läuger, Extended Stribeck curves for food samples, Biosurf. Biotribol. 4, 34 (2018).

[14] M. Diew, A. Ernesto, J. Cayer-Barrioz, and D. Mazuyer, Stribeck and traction curves under moderate contact pressure: From friction to interfacial rheology, Tribol. Lett. 57, 8 (2015).

[15] J. Vicente, J. R. Stokes, and H. A. Spikes The frictional properties of Newtonian fluids in rolling-sliding soft-EHL contact, Tribol. Lett. 20, 273 (2005).

[16] S. Smith, D. Dowson, and A. A. J. Goldsmith, The effect of femoral head diameter upon lubrication and wear of metalmetal total hip replacements, Proceedings of the Institution of Mechanical Engineers, Part H (Journal of Engineering in Medicine) 215, 161 (2001).

[17] J. N. Israelachvili, Y. Min, M. Akbulut, A. Alig, G. Carver, W. Greene, K. Kristiansen, E. Meyer, N. Pesika, K. Rosenberg, and H. Zeng Recent advances in the surface forces apparatus (SFA) technique, Rep. Prog. Phys. 73, 036601 (2010).
[18] T.-D. Li and E. Riedo, Nonlinear Viscoelastic Dynamics of Nanoconfined Wetting Liquids, Phys. Rev. Lett. 100, 106102 (2008).

[19] T. G. Mezger, The Rheology Handbook (Vincentz Network, Hanover, 2006).

[20] H. Bruus, Theoretical Microfluidics (2008).

[21] P. L. Kapitza, The hydrodynamic theory of lubrication in the presence of rolling, Zh. Tekh. Fiz. 25, 747 (1955).

[22] D. E. Brewe, B. J. Hamrock, and C. M. Taylor, Effect of geometry on hydrodynamic film thickness, J. Lubr. Technol. 101, 231 (1979).

[23] See Supplemental Material at http://link.aps.org/ supplemental/10.1103/PhysRevLett.126.044301 for the derivation of the normal- and friction force and the accompanying dimensionless functions.

[24] From Eq. (12) in [22], rewritten in the shape of our Eq. (2), taking $\alpha=1, \phi=3 / 5$, and $L(\alpha=1)=1.7305$ as found in Table II of [22].

[25] D. Petrova, B. Weber, C. Allain, P. Audebert, C. H. Venner, A. M. Brouwer, and D. Bonn Fluorescence microscopy visualization of the roughness-induced transition between lubrication regimes, Sci. Adv. 5, eaaw4761 (2019). 